\title{
Role of the PI3K/Akt signaling pathway in liver ischemia reperfusion injury: a narrative review
}

\author{
Mengqin Wang ${ }^{1,2,3,4,5}$, Ji Zhang ${ }^{6,7,8}$, Nianqiao Gong ${ }^{1,2,3,4,5}$ \\ ${ }^{1}$ Institute of Organ Transplantation, Tongji Hospital Affiliated to Tongji Medical College, Huazhong University of Science and Technology, Wuhan, \\ China; ${ }^{2}$ Key Laboratory of Organ, Wuhan, China; ${ }^{3}$ Transplantation, Ministry of Education, Wuhan, China; ${ }^{4}$ NHC Key Laboratory of Organ \\ Transplantation, Wuhan, China; ${ }^{5}$ Key Laboratory of Organ Transplantation, Chinese Academy of Medical Sciences, Wuhan, China; ${ }^{6}$ Department \\ of Urology, the First Affiliated Hospital of Anhui Medical University, Hefei, China; ${ }^{7}$ Institute of Urology, Anhui Medical University, Hefei, China; \\ ${ }^{8}$ Anhui Province Key Laboratory of Genitourinary Diseases, Anhui Medical University, Hefei, China \\ Contributions: (I) Conception and design: All authors; (II) Administrative support: N Gong; (III) Provision of study materials or patients: None; (IV) \\ Collection and assembly of data: None; (V) Data analysis and interpretation: None; (VI) Manuscript writing: All authors; (VII) Final approval of \\ manuscript: All authors. \\ Correspondence to: Nianqiao Gong, MD. Institute of Organ Transplantation, Tongji Hospital Affiliated to Tongji Medical College, Huazhong \\ University of Science and Technology, Wuhan 430030, China. Email: nqgong@tjh.tjmu.edu.cn.
}

Objective: To explore the role of phosphatidylinositol-3-kinase/protein kinase B and alpha serine/ threonine protein kinase PI3K/PKB (also known as PI3K/Akt) signaling pathway in liver ischemia reperfusion injury.

Background: The PI3K/Akt signaling pathway is one of the major signal transduction pathways that regulates numerous cellular activities in vivo. The main functions of this pathway include induction of stem cell differentiation and metastasis, promotion of cell proliferation, inhibition of apoptosis, and regulation of tissue inflammation, tumor growth, and invasion. Liver ischemia reperfusion injury is an inevitable clinical problem that can occur during liver transplantation, liver resection, and various circulatory shock events, and it is one of the primary reasons for postoperative liver dysfunction, and poor disease outcome and patient prognosis. In recent years, it has been found that PI3K/Akt signaling pathway is closely related to liver ischemia reperfusion injury.

Methods: In this review, a large number of relevant literatures were collected to explain the biological basis of PI3K/Akt signaling pathway and its role in liver ischemia reperfusion injury. The review was based on a PubMed search using the terms "liver ischemia reperfusion injury", "PI3K/Akt signaling pathway", and "PI3K/Akt signaling pathway AND liver ischemia reperfusion injury", so as to understand the complex interaction between them.

Conclusions: Activated PI3K/Akt signaling pathway can exert anti-inflammatory, antioxidant stress, antiapoptosis and autophagy regulation effects through downstream related targeted pathways and proteins, thus alleviating liver ischemia-reperfusion injury. Therefore, the regulation of PI3K/Akt signaling pathway is expected to become an effective targeted pathway for clinical prevention and alleviation of liver ischemia reperfusion injury.

Keywords: Phosphatidylinositol-3-kinase/protein kinase B (PI3K/Akt); liver ischemia reperfusion injury (LIRI); oxidative stress; apoptosis; autophagy

Submitted Nov 09, 2021. Accepted for publication Dec 10, 2021.

doi: 10.21037/apm-21-3286

View this article at: https://dx.doi.org/10.21037/apm-21-3286 


\section{Introduction}

Liver ischemia reperfusion injury (LIRI) commonly occurs during liver surgery, hemorrhagic shock, and other processes, and can lead to severe metabolic disorders, inflammatory immune responses, oxidative stress injury, and cell apoptosis. Studies have indicated that the pathogenesis of LIRI involves a number of different mechanisms, including oxidative stress, inflammatory responses, apoptosis, liver Kupffer cell activation and neutrophil aggregation, vascular cell adhesion molecule overexpression, autophagy, and calcium overload $(1,2)$. The phosphatidylinositol-3-kinase/protein kinase B (PI3K/ Akt) signaling pathway is one of the important pathways regulating various cellular functions and plays an important role in numerous physiological processes and pathological responses. The PI3K/Akt signaling pathway is activated during the early stage of LIRI, where it plays anti-apoptosis, anti-inflammatory, anti-oxidative stress, and autophagy regulation roles. In this review, the functional characteristics of this pathway and its role in the various pathogenesis processes of LIRI are discussed.

We present the following article in accordance with the Narrative Review reporting checklist (available at https://apm. amegroups.com/article/view/10.21037/apm-21-3286/rc).

\section{PI3K/Akt signaling pathway}

\section{General information regarding PI 3 K}

PI3K has dual activities of lipid kinase and protein kinase, which belongs to the lipid kinase family of phosphorylated phosphatidylinositol, a component of eukaryotic cell membranes (3). Based on sequence homology and lipid substrate characteristics, PI3K is divided into three categories (class I, II, and III), as shown in Figure 1, where the PI3K I class has been the most thoroughly studied (4). The PI3K I class exists as dimers that are composed of a regulatory subunit and a catalytic subunit. According to their different molecular structures, PI3K proteins can be divided into groups A and B $(5,6)$. Class IA PI3K consists of a heterodimer between the P85 regulatory subunit and the P110 catalytic subunit (7). In mammals, the P85 subunits are further subdivided into $\alpha, \beta$, and $\gamma$ types that are encoded by the PI3K regulatory subunit (PIK3R) 1, PIK3R2 and PIK3R3, respectively. The specific functions of the P85 subunit include receptor binding, enzyme activation, and localization (8-11). The p110 subunit consists of $\alpha, \beta$, and $\delta$ types that are encoded by the PI3K catalytic subunits (PIK3C) A, PIK3CB, and PIK3CD, respectively (12). P110 $\alpha$ and p110 $\beta$ primarily affect cell proliferation and insulin signaling in various tissues, while p110 is found only in leukocytes and is involved in immune function and inflammation $(9,13)$. Class IB PI3K consists of the regulatory $\mathrm{P} 101$ subunit and the catalytic P110 $\gamma$ subunit. P110 $\gamma$ is primarily expressed in white blood cells, and its expression is reduced in the heart, pancreas, liver, and skeletal muscle. The P85 regulatory subunit of class IA PI3K consists of the SH3 domain, the homology of the Rho binding domain/breakpoint cluster, the C-terminal $\mathrm{SH} 2$ domain, and the junction region $(6,9,14)$. The PI3K II class does not possess a regulatory subunit and instead only contains a catalytic subunit. This protein exists as three subtypes $(\alpha, \beta$, and $\gamma)$ that contain proline-rich domains, RAS binding domains, HR3 domains, HR2 domains, HR1 domains, PX domains, and C2 domains (6,15-17). Vps34 is the only member of the class III PI3K family, and this protein is a human homologous yeast gene product. Human $\mathrm{PI} 3 \mathrm{~K}$ is a serine/threonine kinase that is also known as Vps34 (9). Vps34 is a heterodimer formed by the regulatory subunit of P150 and the catalytic subunit of nutmeg acylation of P100, which phosphorylates PI to PI3P and mediates autophagy and protein synthesis under nutritional stress $(9,18,19)$. Other studies have pointed out that although PI3P is widely present, its level does not change when cells are stimulated, so it is considered that PI3K class iii may be a housekeeping kinase that does not play a role in signal transduction (20).

\section{General information regarding Akt}

Akt is a serine/threonine kinase that functions as the central mediator of the PI3K pathway and plays a key role in numerous cellular processes, including glucose metabolism, apoptosis, cell proliferation, transcription, and cell migration (21). There are three subtypes of Akt that include $\mathrm{PKB} \alpha$ (Akt1), PKB $\beta$ (Akt2), and PKB $\gamma$ (Akt3) $(22,23)$, as shown in Figure 2. Akt1 is widely expressed in a number of tissues, while Akt2 is primarily expressed in insulin-sensitive tissues such as skeletal muscle, adipose tissue, and liver, and Akt3 is predominantly expressed in the testes and brain. Akt consists of a $\mathrm{pH}$ domain, an intermediate kinase domain, and a regulating carboxyl terminal domain in which the $\mathrm{pH}$ domain regulates Akt transposition (24). Akt is activated through two key phosphorylation processes. PIP3 as an intracellular second messenger, and the structure of the $\mathrm{pH}$ domain within Akt can facilitate binding to PIP3 to cause 


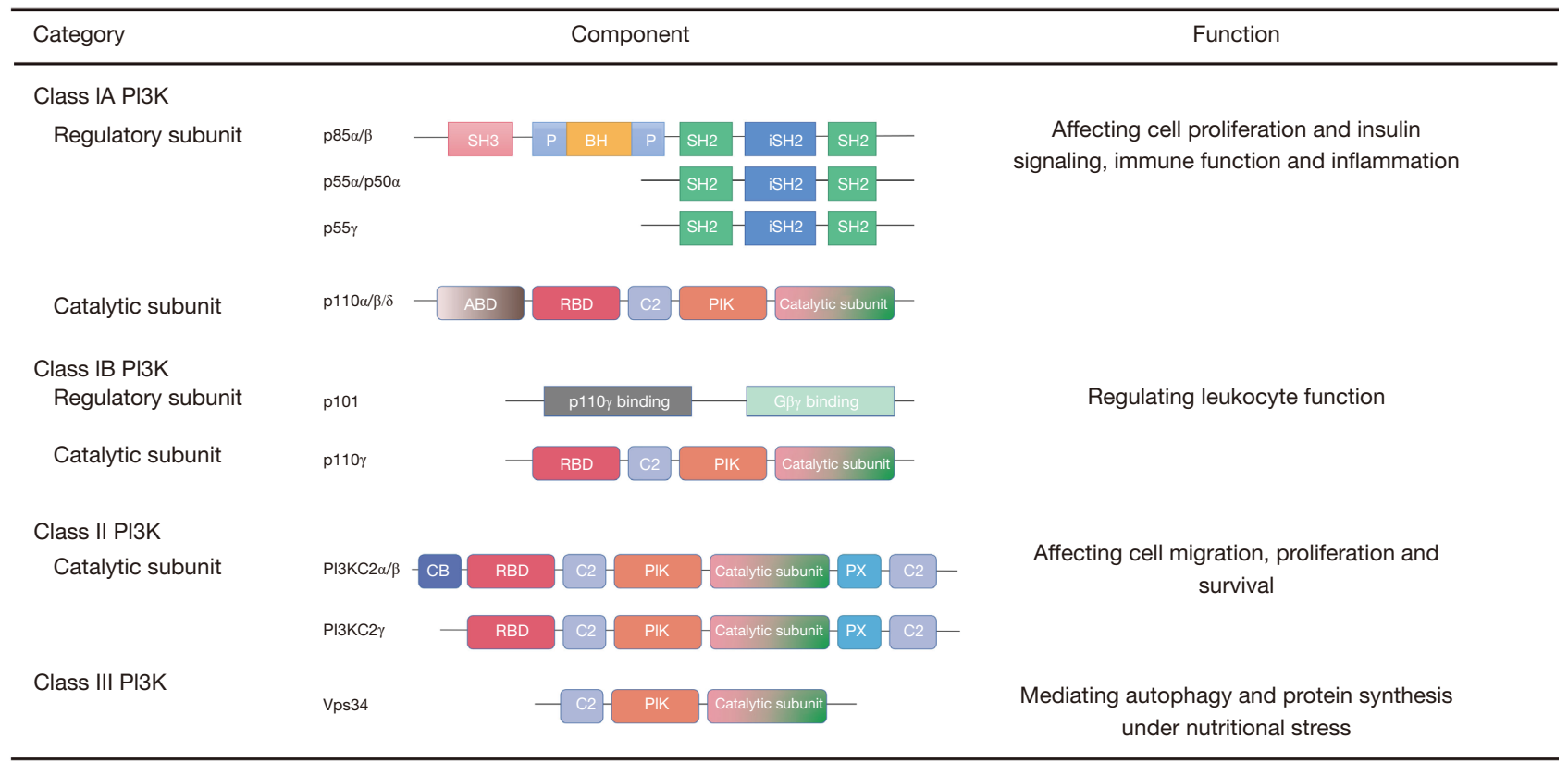

Figure 1 Structural overview of each type of PI3K. SH3, Src homology 3; P, proline-rich region; BH, breakpoint cluster region homology; $\mathrm{SH}$ 2, Src homology 2; iSH2, interSH2; ABD, adapter binding domain; RBD, Ras-binding domain; PI3K, phosphatidylinositol-3-kinase; CB, clathrin binding domain; PX, phox homology.

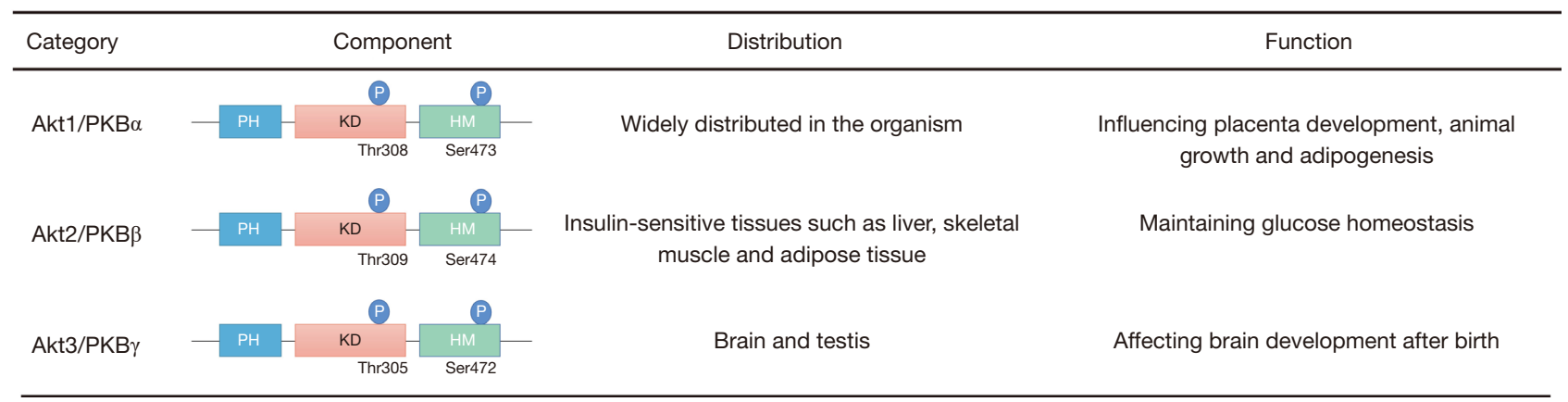

Figure 2 The structure and function of each type of Akt. PH, pleckstrin homology domain; KD, kinase domain; HM, hydrophobic motif.

a conformation change in AT to expose the Ser473 and Thr308 sites to phosphoinositide dependent protein kinase 1 (PDK1) phosphorylation of the kinase Thr308 structure This further facilitates the Akt activation process, where mammalian target of rapamycin (mTOR) compound 2 (mTORC2) can initiate Ser473 phosphorylation facilitates additional adjustment structure of the domain at the terminus of the carboxyl region, thereby fully activating Akt (25). Similar phosphorylation events were observed on the corresponding residues of Akt2 (Thr309 and Ser474) and Akt3 (Thr305 and Ser472). Phosphorylation of both residues is necessary for maximum activation of $\mathrm{Akt}$, and protein 2A phosphatase ( $\mathrm{PP} 2 \mathrm{~A})$ and leucine-rich repeat protein phosphatase function to dephosphorylate Thr308 and Ser473 of Akt, respectively, thus leading to Akt inactivation (26).

\section{Types and stages of LIRI}

LIRI is mainly divided into two types: (I) warm ischemia reperfusion injury, which occurs during liver resection, shock or trauma caused by various reasons, and can lead 
to liver or even multiple organ failure; (II) cold ischemia reperfusion injury usually occurs during organ preservation and transplantation. Historically, it has been believed that liver cells are more sensitive to warm ischemia reperfusion, while liver sinusoidal endothelial cells (LSEC) are more sensitive to cold ischemia reperfusion. However, limitations on damage to specific cell types are rare under pathophysiological conditions $(1,27)$.

LIRI includes two stages of ischemia and reperfusion: ischemia leads to decreased intracellular 5'-adenosine triphosphate (ATP) concentration, ATP-dependent $\mathrm{Na}^{+}$/ $\mathrm{K}^{+}$pump failure, cell edema, and increased cytoplasmic calcium concentration, all of which leads to cell damage. Liver reperfusion enhances cellular damage through oxidative stress and inflammatory response. The initial stage of ischemia reperfusion injury (within 2 hours after reperfusion) involves Kupffer cells releasing reactive oxygen species (ROS) and pro-inflammatory mediators such as tumor necrosis factor (TNF)- $\alpha$, interleukin (IL)-6, IL-1, and arachidonic acid. ROS may lead to lipid peroxidation, mitochondrial permeability conversion, signal transduction pathway activation, caspase activation, hepatocyte and endothelial cell necrosis and apoptosis. Late stages (6-48 $\mathrm{h}$ after reperfusion) are characterized by neutrophilmediated inflammatory responses. Cytokines, chemokines, and complement factors recruit neutrophils into the liver, where they damage liver cells by releasing ROS or proteases. Endothelin-1 (ET-1)-mediated vasoconstriction and increased platelet aggregation lead to microcirculation disorders and aggravate liver injury. Although there is a chronological sequence of events that occur during LIRI, these events may overlap or occur simultaneously in the body (28-30).

\section{PI3K/Akt signaling pathway and LIRI}

\section{PI3K/Akt signaling pathway and anaerobic metabolism and acidosis}

When the liver is in the ischemic stage, the tissue is in a state of ischemia and hypoxia, and cell normal redox process is blocked, the ATP in the cell was quickly consumption, anaerobic glycolysis increase, lead to the accumulation of lactic acid and ketone body acidic metabolites, and mitochondrial oxidative phosphorylation function decline, the organization cell $\mathrm{pH}$ decline, metabolic acidosis. After refluxes, the $\mathrm{pH}$ value returned to normal, and a large number of $\mathrm{pH}$-dependent proteases, phospholipases and other activities increased, but aggravated tissue and organ damage, resulting in LIRI, which is one of the most common mechanisms of LIRI (27).

Rafiee et al. found that acidic microenvironment can activate the PI3K/Akt signaling pathway and significantly enhance the phosphorylation of Akt (31). After stimulated by factors such as hypoxia and acidic environment, the regulatory subunit $\mathrm{P} 85$ of $\mathrm{PI} 3 \mathrm{~K}$ relieved the inhibition of catalytic pressure group P110, and the spatial conformation of PI3K dimer changed and was then activated, which promoted the formation of phosphatidylinositol 3,4,5-triphosphate (PIP3) on the membrane of PI3K. Signaling proteins are then recruited to regulate various intracellular activities such as cell survival, proliferation and differentiation (15).

Metabolic acidosis occurs during LIRI, and the acidic microenvironment affects the activity and function of lymphocytes. Studies have found that regulatory $\mathrm{T}$ cells (Tregs) can significantly relieve the inflammatory response caused by LIRI, and have a certain protective effect on injured organs (32-34). The acidic microenvironment of LIRI down-regulated the function of Tregs and promoted the apoptosis of Tregs. It is known that V-ATPase located in internal acidic vesicles and plasma membrane plays a role in regulating $\mathrm{pH}$ gradient across membrane $(35,36)$. Tregs expressed more V-ATPase in acidic microenvironment. Some scholars have found that omeprazole can inhibit $\mathrm{PI} 3 \mathrm{~K} / \mathrm{Akt} / \mathrm{mTOR}$ signaling pathway by reducing v-ATPase expression, improve the induction efficiency of Tregs in acidic microenvironment, and then improve LIRI acidic microenvironment and reduce LIRI $(37,38)$.

\section{PI3K/Akt signaling pathway and oxidative stress}

Due to ischemia and hypoxia that occur during LIRI, oxygen free radicals are stimulated through xanthine oxidase system, phagocyte system and mitochondrial respiratory chain during reperfusion, resulting in increased production of ROS and insufficient scavenging capacity, thus resulting in liver cell damage. Increased ROS can damage liver cells by generating a variety of lipid peroxides that damage the structure and function of LSEC, cause DNA mutations in the nucleus of liver parenchyma tissues, inhibit oxidative phosphorylation of mitochondria, and cause microcirculation disorders (2,39-41).

PI3K is a redox sensitive kinase that can be activated by changes in intracellular ROS levels (42). During the process of LIRI, the increase in intracellular ROS and 
oxidative stress levels cause compensatory activation of the PI3K/Akt signaling pathway. The activated PI3K/ Akt signaling pathway promotes the generation of heme oxygenase-1 (HO-1) by upregulating the expression of $\mathrm{Nrf2}$, thus alleviating oxidative stress injury during LIRI (43-47). In addition, activation of PI3K/Akt pathway can activate endothelial nitric oxide synthase (eNOS) in vascular endothelial cells, increase the formation of nitric oxide (NO), and further improve LIRI (48-50).

\section{PI3K/Akt signaling pathway and inflammatory response}

In the early stages of ischemia reperfusion, Kupffer cells are activated, which, in addition to producing a large number of ROS, increases the release of pro-inflammatory chemokines and cytokines that promote and amplify subsequent neutrophil-mediated inflammatory responses $(51,52)$. Stimulated by pro-inflammatory factors and chemokines at the early stage, neutrophils adhere to and aggregate in the liver space. These neutrophils release a large number of proteases (e.g., collagenase, elastase, cathepsin G) that act on cell membranes and matrix components, thereby promoting cellular degradation (53). Neutrophils also drive inflammation of liver damage by releasing inflammatory mediators, including proteolytic enzymes, lipocalin 2, arachidonic acid metabolites, and ROS (54); In response to macl-1 adhesion, neutrophils release myeloperoxidase (MPO) and proteases through NADPH oxidase and threshing, leading to superoxide formation. It can directly cause liver endothelial injury or indirectly induce tissue injury by triggering other inflammatory mediators (55).

During LIRI, Kupffer cell activation significantly increases the expression of high-mobility group B1 (HMGB1), and this activates Toll-like receptor 4 (TLR4) to trigger the recruitment of MyD88 and subsequently promote the activation of downstream signaling pathways, ultimately leading to significant increases in NF- $\kappa \mathrm{B}$, TNF- $\alpha$, and IL-6 (56). PI3K can inhibit the activation of $\mathrm{NF}-\kappa \mathrm{B}$ and its downstream pro-inflammatory cytokines by inactivating TLR4 signaling through preventing the recruitment of Toll-IL-1 resistance domain junction protein (TIRAP) to the cell membrane, thus alleviating LIRI (57-59). Blocking of PI3K/Akt leads to increased transcription of NF- $\kappa \mathrm{B}$ and the release of TNF- $\alpha$, IL$1 \beta$, and IL- 6 to ultimately exacerbate LIRI (60). Shen et al. determined that activation of the PI3K/Akt signaling pathway during LIRI increased the expression of IL-4 and IL-10, decreased the expression of IL- $1 \beta$ and TNF- $\alpha$, and reduced the liver inflammatory response (50). Our recent study found that methyl eugenol can reduce LIRI by activating the PI3K/Akt signaling pathway, inhibiting the expression of inflammatory factors and reducing the apoptosis rate (61).

\section{PI3K/Akt signaling patbway and apoptosis}

Apoptosis is closely related to LIRI, and apoptosis of hepatocytes and hepatic sinusoidal endothelial cells is an important cause of LIRI. The process of apoptosis is extremely complex, and the generation of oxygen free radicals, cell energy metabolism disorder, intracellular calcium overload, the activation of cytokines and caspase and B lymphocyte tumor-2 (Bcl-2) family gene expression is closely related to the mechanism disorder will induce a variety of diseases. Disorders of the mechanisms underlying apoptosis, including the death receptor pathway, the mitochondrial pathway, the perforin/granzyme pathway, and the endoplasmic reticulum pathway, may induce various diseases. The mitochondrial pathway, also known as endogenous apoptosis pathway, is the most important pathway for mediating apoptosis. When cells receive a variety of endogenous apoptosis signal stimulations, the Bcl-2 protein regulates mitochondrial permeability by irreversibly opening the mitochondrial permeability transition pore (mPTP), ultimately resulting in clearance of cytochrome $\mathrm{C}$ into the cytoplasm. Apoptosis protein activated factor 1 (Apaf-1) then interacts with this released cytochrome C to facilitate increased levels of the caspase 9 precursor that then becomes active through an auto-cleavage process. Activated caspase 9 activates a downstream caspase cascade that is mediated by caspase 3 to induce apoptosis $(62,63)$.

The PI3K/Akt signaling pathway is a classical antiapoptotic pathway. When the PI3K/Akt signaling pathway is activated, Akt inhibits caspase 3-mediated cell death by phosphorylating Bcl-2/Bcl-XL-associated death promoter (Bad). Concurrently, the depolymerization of Bcl2 with phosphorylated Bad further promotes cell survival. Studies have demonstrated that activation of the PI3K/Akt/ mTOR signaling pathway can significantly increase Bcl2 protein levels, reduce the expression of Bax and caspase 3 proteins, protect cells from apoptosis, and significantly improve LIRI (3,27,64-68).

\section{PI3K/Akt signaling pathway and autophagy}

Autophagy is a lysosomal dependent catabolic process that 
plays an important role in regulating cell homeostasis by degrading damaged organelles and misfolded proteins. Autophagy is not only important for the dynamic balance of proteins, but it also acts a quality control system under internal and external stress conditions and is closely related to the occurrence and development of a variety of physiological processes (such as nutritional deficiency, ischemia, hypoxia, and others) and diseases (such as neurodegenerative diseases, immune diseases, and others). During LIRI, autophagy can be activated by adverse factors such as reduced ATP synthesis, elevated production of ROS, and calcium overload. Mitochondrial autophagy can rapidly remove the aging and damaged mitochondria and excess oxygen free radicals through the lysosomal mechanism to thus maintain the normal function of mitochondria and alleviate LIRI. However, when the content of oxygen free radicals and calcium ions exceeds the clearance rate of autophagy, autophagy cannot clear all damaged mitochondria. This eventually leads to irreversible cell death (69). Currently, it remains undetermined whether autophagy plays a protective or injurious role in LIRI. It should be noted that class I PI3K and class III PI3K play different roles in autophagy regulation. Class I PI3K inhibits autophagy through PI3K/Akt/mTORC1 pathway, while class III PI3K enhances autophagy by inducing Beclin1 (70).

It has been demonstrated that Akt regulates autophagy levels by directly phosphorylating and inhibiting adenosine monophosphate-activated protein kinase (AMPK)-mediated phosphorylation of tuberous sclerosis complex 2 (TSC2), thus inducing complete inhibition of TSC2 and activation of mTOR (71). Yang et al. found that downregulation of the PI3K/Akt/mTOR signaling pathway resulted in increased expression of Beclin-1, Atg7, and LC3 and decreased expression of $\mathrm{p} 62$, thus indicating that autophagy can be properly enhanced to protect the liver from ischemia-reperfusion injury (72). A recent study showed that octreotide and melatonin can enhance autophagy by regulating AMPK/PI3K/Akt/mTOR/ULK1 signaling, and subsequently reduce LIRI (73).

Liu et al. revealed that shikonin treatment could improve LIRI by upregulating the expression of PI3K and p-Akt and inhibiting autophagy. PI3K can upregulate the expression of the Bcl-2 protein, and the Bcl-2/Beclin- 1 complex plays a key role in the regulation of apoptosis and autophagy (74). A recent study demonstrated that upregulation of $\mathrm{Bcl}-2$ could inhibit the disassociation of Beclin-1 and Bcl-2, thus leading to decreased Beclin-1 expression and inhibition of autophagy (75). Various doses of shikonin significantly decreased the expression of LC3 and Beclin- 1 and increased the expression of $\mathrm{P} 62$, thus suggesting that shikonin treatment can inhibit autophagy by activating the PI3K/ Akt pathway and can thereby prevent hepatic ischemia reperfusion injury (64).

\section{PI3K/Akt signaling patbway and heat shock proteins (HSPs)}

HSPs are also known as emergency proteins and exist as a group of structurally highly conserved peptides. HSPs possess a variety of biological activities, and their most important role is to act as molecular chaperones where they aid in facilitating the correct folding of newly synthesized proteins, influence the refolding of proteins destroyed by stress, and maintain the stability of various protein structures. Studies have revealed that there are multiple HSP expressions during LIRI (76,77). HSP70 is the most highly conserved and most important HSP. The expression level of HSP70 in normal cells is typically low. In response to various stimuli such as ischemia, hypoxia, and inflammatory responses, HSP70 can migrate from the cytoplasm to the nucleus and become expressed at high levels. HSP70 possesses immunomodulatory properties, and in combination with IL-2, it can enhance the ability of Tregs to increase the secretion levels of the immunosuppressive cytokines IL-10 and TGF- $\beta$. Human HSP70 enhances the regulatory activity of Tregs primarily through modulating the interaction between TLR2 and T cells, and PI3K/Akt is involved in HSP70-dependent activation of Tregs (78). Zheng et al. revealed that HSP70 can protect the liver from ischemia-reperfusion injury by stimulating the PI $3 \mathrm{~K} / \mathrm{Akt}$ signaling axis to regulate the differentiation of Tregs (79).

\section{PI3K/Akt signaling patbway and non-coding $R N A$}

Non-coding RNA refers to a type of RNA that does not participate in encoding proteins during the process of gene expression transcription and instead plays an important role in the process of transcription and post-transcription modification. During LIRI, the expression of certain non-coding RNAs within cells becomes abnormal, and these non-coding RNAs are primarily involved in cell energy metabolism, apoptosis, autophagy, oxidative stress responses, and inflammation (80).

MicroRNAs (miRNAs) can recognize and target mRNA to regulate the expression of target genes at the 
post-transcriptional or translational level. Studies have demonstrated that miR-494 can upregulate the PI3K/Akt pathway by targeting PTEN to reduce cell apoptosis and improve LIRI in rats (81).

Long non-coding RNAs (lncRNAs) can interact with all types of intracellular macromolecules, including proteins, DNA, and RNA. LncRNA can be used as signal, bait, guide, skeleton, and enhancement molecule to modulate gene expression at the transcriptional and post-transcriptional levels. Cui et al. demonstrated that lncRNA H19 could enhance autophagy-induced hypoxia/reoxygenation injury in liver cancer cells through downregulation of the PI3K/ Akt/mTOR pathway (82).

The main biological function of small interfering RNA (siRNA) is to participate in RNA interference (RNAi). RNAi induces gene silencing by targeting mRNA for degradation or by preventing mRNA from being translated into proteins. During the process of LIRI, siRNA-induced $\beta$-catenin deficiency and STAT3 silencing are used to inhibit PTEN and activate the PI3K/Akt pathway, and this can downregulate the immune function of dendritic cells (DCs), inhibit TLR4-driven inflammatory responses, and reduce cell apoptosis $(83,84)$.

\section{PI3K/Akt signaling patbway and exosomes}

Exosomes are a class of tiny vesicles possessing a lipid bilayer structure and ranging in size from 30 to 150 $\mathrm{nm}$ in diameter. Exosomes can transmit information between cells, and the proteins, DNA, and RNA that they carry can be absorbed by the recipient cells to facilitate specific functions. Studies have revealed that hepatocyte-, DC-, and mesenchymal bone marrow stem cell-derived exosomes can mitigate LIRI through different mechanisms $(79,85,86)$. DC-derived exosomes inhibit the immune inflammatory cascade and reduce inflammation-induced hepatocyte necrosis, and Zheng et al. revealed that DCderived exosomes can activate the PI $3 \mathrm{~K} / \mathrm{Akt} / \mathrm{mTOR}$ signaling pathway in the initial $\mathrm{CD}^{+} \mathrm{T}$ lymphocytes by delivering HSP70 to these lymphocytes. This induced the differentiation of the initial $\mathrm{CD}^{+} \mathrm{T}$ lymphocytes into Tregs and indirectly inhibited the differentiation of the initial $\mathrm{CD}^{+} \mathrm{T}$ lymphocytes into Th17 cells. Amplified Tregs can induce the formation and maintenance of immune tolerance, and at the same time, the reduction of Th17 production can further inhibit the production of immune inflammatory cascade, thus inhibiting the necrosis and apoptosis of liver cells induced by inflammation to ultimately improve LIRI (79).

\section{Conclusion and prospects}

LIRI is a serious clinical problem that is considered to be the potential mechanism underlying the dysfunction and injury of organs other than the liver itself. Recent studies demonstrated that the PI3K/Akt signaling pathway is closely associated with LIRI, and PI3K/Akt signaling can improve LIRI by influencing the anti-oxidative stress responses, anti-inflammatory processes, regulation of immune cells, regulation of autophagy, and reduction of cell apoptosis (as shown in Figure 3).

However, in the process of LIRI, how the PI3K/Akt signaling pathway coordinates the functions of the above pathological phenomena and whether there are interactions between these phenomena still need to be further discussed. In addition, most current studies are only limited to the involvement of PI3K/Akt signaling pathway in LIRI, but how PI3K/Akt signaling pathway is activated during LIRI has not been clarified. In addition, most of the current studies are focused on animal models, and different experimental conditions and modeling will inevitably produce different experimental results. Whether various intervention methods have the same safety and effect in clinical application remains to be further studied.

It is noteworthy that the abnormal activation of PI3K/ Akt signaling pathway is involved in the regulation of tumor cell survival, proliferation, invasion and migration in the process of various tumors. By inhibiting the expression of each molecule in this pathway and blocking this signaling pathway, tumor growth and metastasis can be inhibited and anti-tumor effect can be achieved $(87,88)$. Therefore, PI3K/ Akt will show different effects in different diseases and cells, so clinical intervention of PI3K/Akt signaling pathway needs to be dealt with in combination with the actual situation.

Therefore, in the future, studies on PI3K/Akt signaling pathway in LIRI can focus on the following points: (I) what is the specific molecular mechanism by which PI3K/Akt signaling pathway is activated or inhibited during LIRI? (II) Whether targeted intervention of PI3K/Akt signaling pathway can reduce liver ischemia-reperfusion injury in large animal experiments, such as pigs and monkeys, or even in clinical process? (III) In the prevention and treatment of LIRI, whether there will be adverse reactions after activation of PI3K/Akt signaling pathway, and how to evaluate its safety? (IV) For liver cancer patients, is it 


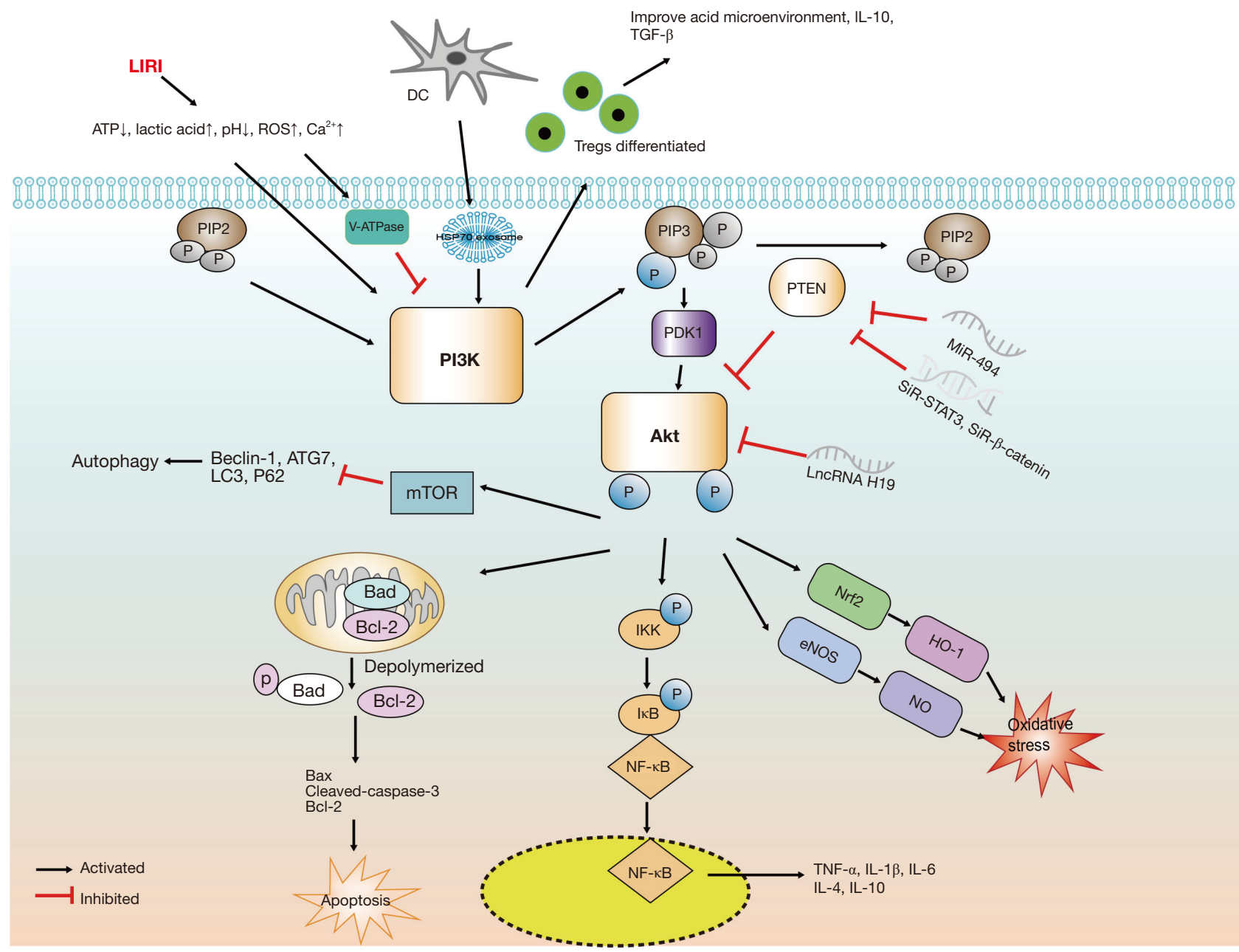

Figure 3 The relationships between liver ischemia reperfusion injury and PI3K/Akt signaling pathway. LIRI, liver ischemia reperfusion injury; ATP, adenosine triphosphate; ROS, reactive oxygen species; DC, dendritic cell; IL, interleukin; TGF, transforming growth factor; PIP2, phosphatidylinositol-4,5-diphosphate; PIP3, phosphatidylinositol-3,4,5-triphosphate; PTEN, phosphatase and tensin homolog; PDK, phosphoinositide dependent protein kinase; PI3K, phosphatidylinositol-3-kinase; Akt, alpha serine/threonine protein kinase; mTOR, mammalian target of rapamycin; ATG, autophagy-related protein; LC3, autophagy microtubule-associated protein light chain $\beta 3$; Bad, Bcl-

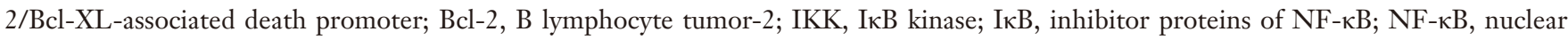
factor- $\mathrm{BB}$; Nrf2, nuclear factor E2 related factor 2; HO-1, heme oxygenase-1;eNOS, endothelial nitric oxide synthase; NO, nitric oxide; TNF, tumor necrosis factor.

feasible to intervene PI3K/Akt signaling pathway to prevent and treat LIRI?

Although there are still some unclarified questions about the effect of PI3K/Akt signaling pathway on LIRI, the PI3K/Akt signaling pathway has the potential to become a target for the prevention and treatment of LIRI, providing a research direction for the prevention and treatment of ischemia reperfusion.

\section{Acknowledgments}

Funding: The present study was supported by the grants from the National Natural Science Foundation of China (grant No. 82170772, 81873623 and 81570678), the Major State Basic Research Development Program of China (grant No. 2013CB530803), the Clinical Research Physician Program of Tongji Medical College, HUST and the Non- 
Profit Central Research Institute Fund of Chinese Academy of Medical Sciences (grant No. 2018PT32018).

\section{Footnote}

Reporting Checklist: The authors have completed the Narrative Review reporting checklist. Available at https:// apm.amegroups.com/article/view/10.21037/apm-21-3286/rc

Peer Review File: Available at https://apm.amegroups.com/ article/view/10.21037/apm-21-3286/prf

Conflicts of Interest: All authors have completed the ICMJE uniform disclosure form (available at https://apm. amegroups.com/article/view/10.21037/apm-21-3286/coif). The authors have no conflicts of interest to declare.

Ethical Statement: The authors are accountable for all aspects of the work in ensuring that questions related to the accuracy or integrity of any part of the work are appropriately investigated and resolved.

Open Access Statement: This is an Open Access article distributed in accordance with the Creative Commons Attribution-NonCommercial-NoDerivs 4.0 International License (CC BY-NC-ND 4.0), which permits the noncommercial replication and distribution of the article with the strict proviso that no changes or edits are made and the original work is properly cited (including links to both the formal publication through the relevant DOI and the license). See: https://creativecommons.org/licenses/by-nc-nd/4.0/.

\section{References}

1. Gracia-Sancho J, Casillas-Ramírez A, Peralta C. Molecular pathways in protecting the liver from ischaemia/ reperfusion injury: a 2015 update. Clin Sci (Lond) 2015;129:345-62.

2. Dar WA, Sullivan E, Bynon JS, et al. Ischaemia reperfusion injury in liver transplantation: Cellular and molecular mechanisms. Liver Int 2019;39:788-801.

3. Fruman DA, Meyers RE, Cantley LC. Phosphoinositide kinases. Annu Rev Biochem 1998;67:481-507.

4. Guo H, German P, Bai S, et al. The PI3K/AKT Pathway and Renal Cell Carcinoma. J Genet Genomics 2015;42:343-53.

5. Graupera $M$, Potente $M$. Regulation of angiogenesis by PI3K signaling networks. Exp Cell Res 2013;319:1348-55.
6. Jean S, Kiger AA. Classes of phosphoinositide 3-kinases at a glance. J Cell Sci 2014;127:923-8.

7. Courtney KD, Corcoran RB, Engelman JA. The PI3K pathway as drug target in human cancer. J Clin Oncol 2010;28:1075-83.

8. Breitkopf SB, Yang X, Begley MJ, et al. A Cross-Species Study of PI3K Protein-Protein Interactions Reveals the Direct Interaction of P85 and SHP2. Sci Rep 2016;6:20471.

9. Engelman JA, Luo J, Cantley LC. The evolution of phosphatidylinositol 3-kinases as regulators of growth and metabolism. Nat Rev Genet 2006;7:606-19.

10. Myers MG Jr, Backer JM, Sun XJ, et al. IRS-1 activates phosphatidylinositol 3'-kinase by associating with src homology 2 domains of p85. Proc Natl Acad Sci U S A 1992;89:10350-4.

11. Siempelkamp BD, Rathinaswamy MK, Jenkins ML, et al. Molecular mechanism of activation of class IA phosphoinositide 3-kinases (PI3Ks) by membranelocalized HRas. J Biol Chem 2017;292:12256-66.

12. Yuan TL, Cantley LC. PI3K pathway alterations in cancer: variations on a theme. Oncogene 2008;27:5497-510.

13. Vivanco I, Sawyers CL. The phosphatidylinositol 3-Kinase AKT pathway in human cancer. Nat Rev Cancer 2002;2:489-501.

14. Schauder C, Ma LC, Krug RM, et al. Structure of the iSH2 domain of human phosphatidylinositol 3-kinase $\mathrm{p} 85 \beta$ subunit reveals conformational plasticity in the interhelical turn region. Acta Crystallogr Sect F Struct Biol Cryst Commun 2010;66:1567-71.

15. Amzel LM, Huang CH, Mandelker D, et al. Structural comparisons of class I phosphoinositide 3-kinases. Nat Rev Cancer 2008;8:665-9.

16. Falasca M, Maffucci T. Role of class II phosphoinositide 3-kinase in cell signalling. Biochem Soc Trans 2007;35:211-4.

17. Gulluni F, De Santis MC, Margaria JP, et al. Class II PI3K Functions in Cell Biology and Disease. Trends Cell Biol 2019;29:339-59.

18. Backer JM. The regulation and function of Class III PI3Ks: novel roles for Vps34. Biochem J 2008;410:1-17.

19. De Santis MC, Gulluni F, Campa CC, et al. Targeting PI3K signaling in cancer: Challenges and advances. Biochim Biophys Acta Rev Cancer 2019;1871:361-6.

20. Bilanges B, Alliouachene S, Pearce W, et al. Vps34 PI 3-kinase inactivation enhances insulin sensitivity through reprogramming of mitochondrial metabolism. Nat Commun 2017;8:1804. 
21. Shariati M, Meric-Bernstam F. Targeting AKT for cancer therapy. Expert Opin Investig Drugs 2019;28:977-88.

22. Huang X, Liu G, Guo J, et al. The PI3K/AKT pathway in obesity and type 2 diabetes. Int J Biol Sci 2018;14:1483-96.

23. Häggblad Sahlberg S, Mortensen AC, Haglöf J, et al. Different functions of AKT1 and AKT2 in molecular pathways, cell migration and metabolism in colon cancer cells. Int J Oncol 2017;50:5-14.

24. Abeyrathna P, Su Y. The critical role of Akt in cardiovascular function. Vascul Pharmacol 2015;74:38-48.

25. Zhang J, Yu XH, Yan YG, et al. PI3K/Akt signaling in osteosarcoma. Clin Chim Acta 2015;444:182-92.

26. Manning BD, Toker A. AKT/PKB Signaling: Navigating the Network. Cell 2017;169:381-405.

27. Nakazato PCG, Victorino JP, Fina CF, et al. Liver ischemia and reperfusion injury. Pathophysiology and new horizons in preconditioning and therapy. Acta Cir Bras 2018;33:723-35.

28. Arii S, Teramoto K, Kawamura T. Current progress in the understanding of and therapeutic strategies for ischemia and reperfusion injury of the liver. J Hepatobiliary Pancreat Surg 2003;10:189-94.

29. Klune JR, Tsung A. Molecular biology of liver ischemia/ reperfusion injury: established mechanisms and recent advancements. Surg Clin North Am 2010;90:665-77.

30. Konishi T, Lentsch AB. Hepatic Ischemia/Reperfusion: Mechanisms of Tissue Injury, Repair, and Regeneration. Gene Expr 2017;17:277-87.

31. Rafiee P, Theriot ME, Nelson VM, et al. Human esophageal microvascular endothelial cells respond to acidic $\mathrm{pH}$ stress by PI3K/AKT and p38 MAPK-regulated induction of Hsp70 and Hsp27. Am J Physiol Cell Physiol 2006;291:C931-45.

32. Feng M, Wang Q, Wang H, et al. Adoptive transfer of hepatic stellate cells ameliorates liver ischemia reperfusion injury through enriching regulatory $\mathrm{T}$ cells. Int Immunopharmacol 2014;19:267-74.

33. Mohr A, Atif M, Balderas R, et al. The role of FOXP3+ regulatory $\mathrm{T}$ cells in human autoimmune and inflammatory diseases. Clin Exp Immunol 2019;197:24-35.

34. Zhu Q, Li C, Wang K, et al. Phosphatase and tensin homolog- -catenin signaling modulates regulatory $\mathrm{T}$ cells and inflammatory responses in mouse liver ischemia/ reperfusion injury. Liver Transpl 2017;23:813-25.

35. Kawai K, Uchiyama M, Hester J, et al. Regulatory T cells for tolerance. Hum Immunol 2018;79:294-303.

36. Nishi T, Forgac $M$. The vacuolar (H+)-ATPases--nature's most versatile proton pumps. Nat Rev Mol Cell Biol
2002;3:94-103.

37. Koltai T. Cancer: fundamentals behind $\mathrm{pH}$ targeting and the double-edged approach. Onco Targets Ther 2016;9:6343-60.

38. Gan X, Zhang R, Gu J, et al. Acidic Microenvironment Regulates the Severity of Hepatic Ischemia/Reperfusion Injury by Modulating the Generation and Function of Tregs via the PI3K-mTOR Pathway. Front Immunol 2020;10:2945.

39. Quesnelle KM, Bystrom PV, Toledo-Pereyra LH. Molecular responses to ischemia and reperfusion in the liver. Arch Toxicol 2015;89:651-7.

40. Prieto I, Monsalve M. ROS homeostasis, a key determinant in liver ischemic-preconditioning. Redox Biol 2017;12:1020-5.

41. Peralta C, Jiménez-Castro MB, Gracia-Sancho J. Hepatic ischemia and reperfusion injury: effects on the liver sinusoidal milieu. J Hepatol 2013;59:1094-106.

42. Ndiaye M, Chataigneau M, Lobysheva I, et al. Red wine polyphenol-induced, endothelium-dependent NOmediated relaxation is due to the redox-sensitive PI3kinase/Akt-dependent phosphorylation of endothelial NOsynthase in the isolated porcine coronary artery. FASEB J 2005; 19:455-7.

43. Deng X, Rui W, Zhang F, et al. PM2.5 induces Nrf2mediated defense mechanisms against oxidative stress by activating PIK3/AKT signaling pathway in human lung alveolar epithelial A549 cells. Cell Biol Toxicol 2013;29:143-57.

44. Ke B, Shen XD, Zhang Y, et al. KEAP1-NRF2 complex in ischemia-induced hepatocellular damage of mouse liver transplants. J Hepatol 2013;59:1200-7.

45. Gao S, Zhang P, Zhang C, et al. Meroterpenoids from Ganoderma sinense protect hepatocytes and cardiomyocytes from oxidative stress induced injuries. Fitoterapia 2018;131:73-9.

46. Li Y, Tong L, Zhang J, et al. Galangin Alleviates Liver Ischemia-Reperfusion Injury in a Rat Model by Mediating the PI3K/AKT Pathway. Cell Physiol Biochem 2018;51:1354-63.

47. Mahmoud AR, Ali FEM, Abd-Elhamid TH, et al. Coenzyme Q10 protects hepatocytes from ischemia reperfusion-induced apoptosis and oxidative stress via regulation of Bax/Bcl-2/PUMA and Nrf-2/FOXO-3/Sirt1 signaling pathways. Tissue Cell 2019;60:1-13.

48. Guo JY, Yang T, Sun XG, et al. Ischemic postconditioning attenuates liver warm ischemia-reperfusion injury through Akt-eNOS-NO-HIF pathway. J Biomed Sci 2011;18:79. 
49. Sabry MM, Ramadan NM, Al Dreny BA, et al. Protective effect of apelin preconditioning in a rat model of hepatic ischemia reperfusion injury; possible interaction between the apelin/APJ system, Ang II/AT1R system and eNOS. United European Gastroenterol J 2019;7:689-98.

50. Shen Y, Shen X, Cheng Y, et al. Myricitrin pretreatment ameliorates mouse liver ischemia reperfusion injury. Int Immunopharmacol 2020;89:107005.

51. Teoh NC, Farrell GC. Hepatic ischemia reperfusion injury: pathogenic mechanisms and basis for hepatoprotection. J Gastroenterol Hepatol 2003;18:891-902.

52. Jaeschke H, Mitchell JR. Use of isolated perfused organs in hypoxia and ischemia/reperfusion oxidant stress. Methods Enzymol 1990;186:752-9.

53. Martins RM, Teodoro JS, Furtado E, et al. Recent insights into mitochondrial targeting strategies in liver transplantation. Int J Med Sci 2018;15:248-56.

54. Qin CC, Liu YN, Hu Y, et al. Macrophage inflammatory protein-2 as mediator of inflammation in acute liver injury. World J Gastroenterol 2017;23:3043-52.

55. El-Benna J, Dang PM, Gougerot-Pocidalo MA, et al. Phagocyte NADPH oxidase: a multicomponent enzyme essential for host defenses. Arch Immunol Ther Exp (Warsz) 2005;53:199-206.

56. Luan X, Liu Y, Li M. The role of CD14 and Toll-like receptor 4 of Kupffer cells in hepatic ischemia-reperfusion injury in rats. Transplant Proc 2012;44:937-41.

57. Guha M, Mackman N. The phosphatidylinositol 3-kinaseAkt pathway limits lipopolysaccharide activation of signaling pathways and expression of inflammatory mediators in human monocytic cells. J Biol Chem 2002;277:32124-32.

58. Díaz-Guerra MJ, Castrillo A, Martín-Sanz P, et al. Negative regulation by phosphatidylinositol 3-kinase of inducible nitric oxide synthase expression in macrophages. J Immunol 1999;162:6184-90.

59. Li X, Wu Y, Zhang W, et al. Pre-conditioning with tanshinone IIA attenuates the ischemia/reperfusion injury caused by liver grafts via regulation of HMGB1 in rat Kupffer cells. Biomed Pharmacother 2017;89:1392-400.

60. Xiao Q, Ye Q, Wang W, et al. Mild hypothermia pretreatment protects against liver ischemia reperfusion injury via the PI3K/AKT/FOXO3a pathway. Mol Med Rep 2017;16:7520-6.

61. Wang M, Zhang J, Zhang J, et al. Methyl eugenol attenuates liver ischemia reperfusion injury via activating PI3K/Akt signaling. Int Immunopharmacol 2021;99:108023.
62. Qi H, Jiang Y, Yin Z, et al. Optimal pathways for the assembly of the Apaf-1.cytochrome c complex into apoptosome. Phys Chem Chem Phys 2018;20:1964-73.

63. Martinou JC, Youle RJ. Mitochondria in apoptosis: Bcl2 family members and mitochondrial dynamics. Dev Cell 2011;21:92-101.

64. Sheng M, Zhou Y, Yu W, et al. Protective effect of Berberine pretreatment in hepatic ischemia/reperfusion injury of rat. Transplant Proc 2015;47:275-82.

65. Mukhopadhyay S, Panda PK, Sinha N, et al. Autophagy and apoptosis: where do they meet? Apoptosis 2014;19:555-66.

66. Liu J, Li J, Tian P, et al. H2S attenuates sepsis-induced cardiac dysfunction via a PI3K/Akt-dependent mechanism. Exp Ther Med 2019;17:4064-72.

67. Rong YP, Huang HT, Liu JS, et al. Protective Effects of Geniposide on Hepatic Ischemia/Reperfusion Injury. Transplant Proc 2017;49:1455-60.

68. Suo L, Kang K, Wang X, et al. Carvacrol alleviates ischemia reperfusion injury by regulating the PI3K-Akt pathway in rats. PLoS One 2014;9:e104043.

69. Cursio R, Colosetti P, Gugenheim J. Autophagy and liver ischemia-reperfusion injury. Biomed Res Int 2015;2015:417590.

70. Heras-Sandoval D, Pérez-Rojas JM, HernándezDamián J, et al. The role of PI3K/AKT/mTOR pathway in the modulation of autophagy and the clearance of protein aggregates in neurodegeneration. Cell Signal 2014;26:2694-701.

71. Hahn-Windgassen A, Nogueira V, Chen CC, et al. Akt activates the mammalian target of rapamycin by regulating cellular ATP level and AMPK activity. J Biol Chem 2005;280:32081-9.

72. Yang J, Chen Q, Tian S, et al. The role of 1,25-dyhydroxyvitamin D3 in mouse liver ischemia reperfusion injury: regulation of autophagy through activation of MEK/ERK signaling and PTEN/PI3K/Akt/ mTORC1 signaling. Am J Transl Res 2015;7:2630-45.

73. Mohamed DZ, El-Sisi AEE, Sokar SS, et al. Targeting autophagy to modulate hepatic ischemia/reperfusion injury: A comparative study between octreotide and melatonin as autophagy modulators through AMPK/PI3K/AKT/ mTOR/ULK1 and Keap1/Nrf2 signaling pathways in rats. Eur J Pharmacol 2021;897:173920.

74. Liu T, Zhang Q, Mo W, et al. The protective effects of shikonin on hepatic ischemia/reperfusion injury are mediated by the activation of the PI3K/Akt pathway. Sci Rep 2017;7:44785. 
75. Qin H, Tan W, Zhang Z, et al. 15d-prostaglandin $\mathrm{J} 2$ protects cortical neurons against oxygen-glucose deprivation/reoxygenation injury: involvement of inhibiting autophagy through upregulation of Bcl-2. Cell Mol Neurobiol 2015;35:303-12.

76. Ma Y, Lu C, Li C, et al. Overexpression of HSPA12B protects against cerebral ischemia/reperfusion injury via a PI3K/Akt-dependent mechanism. Biochim Biophys Acta 2013;1832:57-66.

77. Dai J, Chen L, Qiu YM, et al. Activations of GABAergic signaling, HSP70 and MAPK cascades are involved in baicalin's neuroprotection against gerbil global ischemia/ reperfusion injury. Brain Res Bull 2013;90:1-9.

78. Zanin-Zhorov A, Cohen IR. Signaling via TLR2 and TLR4 Directly Down-Regulates T Cell Effector Functions: The Regulatory Face of Danger Signals. Front Immunol 2013;4:211.

79. Zheng L, Li Z, Ling W, et al. Exosomes Derived from Dendritic Cells Attenuate Liver Injury by Modulating the Balance of Treg and Th17 Cells After Ischemia Reperfusion. Cell Physiol Biochem 2018;46:740-56.

80. Cui Z, Li S, Liu Z, et al. Interferon Regulatory Factor 1 Activates Autophagy to Aggravate Hepatic IschemiaReperfusion Injury by Increasing High Mobility Group Box 1 Release. Cell Physiol Biochem 2018;48:328-38.

81. Su S, Luo, Liu X, et al. miR-494 up-regulates the PI3K/ Akt pathway via targetting PTEN and attenuates hepatic ischemia/reperfusion injury in a rat model. Biosci Rep

Cite this article as: Wang M, Zhang J, Gong N. Role of the $\mathrm{PI} 3 \mathrm{~K} /$ Akt signaling pathway in liver ischemia reperfusion injury: a narrative review. Ann Palliat Med 2022;11(2):806-817. doi: 10.21037/apm-21-3286
2017;37:BSR20170798.

82. Cui C, Li Z, Wu D. The long non-coding RNA H19 induces hypoxia/reoxygenation injury by up-regulating autophagy in the hepatoma carcinoma cells. Biol Res 2019;52:32.

83. Ke B, Shen XD, Kamo N, et al. $\beta$-catenin regulates innate and adaptive immunity in mouse liver ischemia-reperfusion injury. Hepatology 2013;57:1203-14.

84. Ke B, Shen XD, Ji H, et al. HO-1-STAT3 axis in mouse liver ischemia/reperfusion injury: regulation of TLR4 innate responses through PI3K/PTEN signaling. J Hepatol 2012;56:359-66.

85. Du Y, Li D, Han C, et al. Exosomes from Human-Induced Pluripotent Stem Cell-Derived Mesenchymal Stromal Cells (hiPSC-MSCs) Protect Liver against Hepatic Ischemia/ Reperfusion Injury via Activating Sphingosine Kinase and Sphingosine-1-Phosphate Signaling Pathway. Cell Physiol Biochem 2017;43:611-25.

86. Nojima H, Freeman CM, Schuster RM, et al. Hepatocyte exosomes mediate liver repair and regeneration via sphingosine-1-phosphate. J Hepatol 2016;64:60-8.

87. Yang Q, Jiang W, Hou P. Emerging role of PI3K/AKT in tumor-related epigenetic regulation. Semin Cancer Biol 2019;59:112-24.

88. Spangle JM, Roberts TM, Zhao JJ. The emerging role of PI3K/AKT-mediated epigenetic regulation in cancer. Biochim Biophys Acta Rev Cancer 2017;1868:123-31. 